\title{
AGENT-BASED WHEAT SIMULATION MODEL COOPERATION RESEARCH
}

\author{
Shengping Liu ${ }^{1}$, Yeping Zhu ${ }^{1, *}$ \\ ${ }^{1}$ Agricultural Information Institute, Chinese Academy of Agricultural Sciences, Beijing, \\ China, 100081; \\ * Corresponding author, Address: Library 311, Agricultural Information Institute, Chinese \\ Academy of Agricultural Sciences, No.12 Zhongguancun South Street, Beijing, 100081, P. \\ R. China, Tel:+86-10-68919652-2342, Fax:+86-10-68919886-2339, Email: zhuyp@mail. \\ caas.net.cn
}

Abstract: Cooperative multi-agent systems (MAS) are ones in which several agents attempt, through their interaction, to jointly solve tasks or to maximize utility. Due to the interactions among the agents, multi-agent problem complexity can rise rapidly with the number of agents or their behavioral sophistication. This paper propose a kind of agent-based cooperation design thought and the realization for wheat simulation model, hangs together the growth model and knowledge model of wheat, realize organic coupling and integration between the function of forecast and decision-making.

Keywords: Multi-agent System, Wheat Simulation Model, Cooperation

\section{INTRODUCTION}

Agriculture system is complicated \& open, since each element within system is highly alternant and cooperative. Applying modern information technology to agriculture production has facilitated the realization of agriculture modernization. As an important aspect, Crop Simulation Model technology is applied to crop production management. There have been lots of outcomes through over 40 years' development, such as CERES 、 GROPGRO. Meanwhile, in order to provide much better technology for agriculture production and decision-making, many researchers are trying to

Please use the following format when citing this chapter:

Liu, S. and Zhu, Y., 2009, in IFIP International Federation for Information Processing, Volume 294, Computer and Computing Technologies in Agriculture II, Volume 2, eds. D. Li, Z. Chunjiang, (Boston: Springer), pp. 1281-1289. 
combine Crop Simulation Model technology with other new technology as well, such as Expert System, Visualization technology, Artificial Intelligence technology, Network technology.

As the same time as information technology successfully applied to agriculture, Agent Theory and Technology, as a part of distributed artificial intelligence, has developed rapidly since it arose from 1970s, it is a popular direction of artificial intelligence now (CHEN Ying-chun, 2003). MultiAgent Technology currently provided new methods and tools for solving complicated problems decision, and for setting up distributed, intelligent, integrated, and man-machine harmony decision making supporting system(Wooldridge, et al. 2000). MAS (Multi-Agent System) plays significant roles in plenty of application fields, such as Industry (Process Control, Remote Telecommunication, Air Traffic Control and Transportation System), Commerce (Information filtration, Information Collection, Ebusiness, Business Process Management), Entertainment (Games, Interactive theater and cinema), Medical Treatment (Remote Medical Treatment and Health Care) and Education (ZHU Ye-ping, et al. 2005).

Current agriculture information system, such as Crop simulation model management system, Expert System, Database, Knowledge Model System, they are independent and unilateral since they aim at solving limited problems. Self-restraint, openness, Interactivity and collaboration are key characters of Agent System (DeLoach, 2004). If we apply Multi-Agent technology to agriculture decision system development, we can reuse and share existed resource, exerting advantages of original system, as well as achieving functional organic coupling and integrated coordination, then improving accuracy and overall of management decision (F. Bousquet and et al. 2002). Building crop management cooperation decision system based on Agent technology, implementing crop information share, software share, cooperation work and group decision support under distributed environment, which will enhance the usage of information, fully exerting efficiency of network, achieving several computers cooperative running (DING Wei-long, 2005).

Meanwhile, it shall react, consult and solve the emergency during crop production, and offer new methods and technology for agriculture production management and environment protection harmony development.

By analyzing characters of Agent, taking advantage of its cooperative work and high intelligent, and combining wheat growth simulation model and wheat growth management knowledge model, then developing wheat production management cooperation decision system based on Agent, in order to realize forecast and decision function organic coupling and integration. 


\section{AGENT-BASED WHEAT SIMULATION MODEL COLLABORATIVE DECISION SYSTEM ANALYSIS AND DESIGN}

Multi-Agent System Analysis and Design is the key of agent technology application, which is not widely used in agent-oriented software engineering field. Now the main modeling methods include AAII modeling methodology, Gaia way, Agent UML methods and $\mathrm{Z}$ language method, and so on (N.HARNOS. 2006). Object-oriented technology and agent-oriented is inextricably linked to each other, at present object-oriented technology has relatively mature development and a lot of practical methods and tools. Visualization tool UML is a excellent object-oriented approach and UMLbased multi-agent modeling technology is more and more recognized (WENG Wen-yong, and et al. 2004).

Based UML visual modeling technology, form the point of view of solving agriculture system problems this paper realizes agent-based wheat model collaborative decision system analysis and design.

\subsection{Main system function design}

Main system function includes cultivation design, real-time growth simulation, dynamic management control, expert knowledge advisory, data query maintenance, system manage.

Cultivation design function is mainly based on the weather, soil, species and other data of the decision-making point and operates wheat knowledge manage model and wheat growth model so that they cooperate with each other. Finally we build a seemly cultivation program which includes Variety choice, seedtime, seed density and quantity, fertilizer operation and water operation.

Growth simulation forecasting system is based on data and cultivation program. Through operating growth simulation model it can forecast wheat growth dynamic feature which includes wheat daily organ dry weight, root density index, leaf area index, water use and stress, nitrogen use and stress.

Dynamic management control function uses suitable dynamic growth targets (such as leaf age, leaf area index, dry matter accumulation and source library target, etc.) as standard expert curve. When the growth shape which is forecasted by growth simulation model significantly deviates from the shape of the standard expert curve, system analysis the cause and recommend an appropriate control measures (such as irrigation, fertilization, etc.) and the regulation period, at the same time, system revise a suitable growth index and let wheat growth in the light of standard expert curve. Finally system output specific control measure, forecasting wheat growth dynamics and yield components. 
Data manage function mainly includes nationwide weather, soil and variety data of decision point. It could not only query and extract required data in database but also add and modify data according to the need.

Expert knowledge advisory includes various kinds of knowledge of wheat growth and development process, which from variety choice to cultivation techniques, irrigation and fertilization, diagnosis and extermination of plant disease and insect pest.

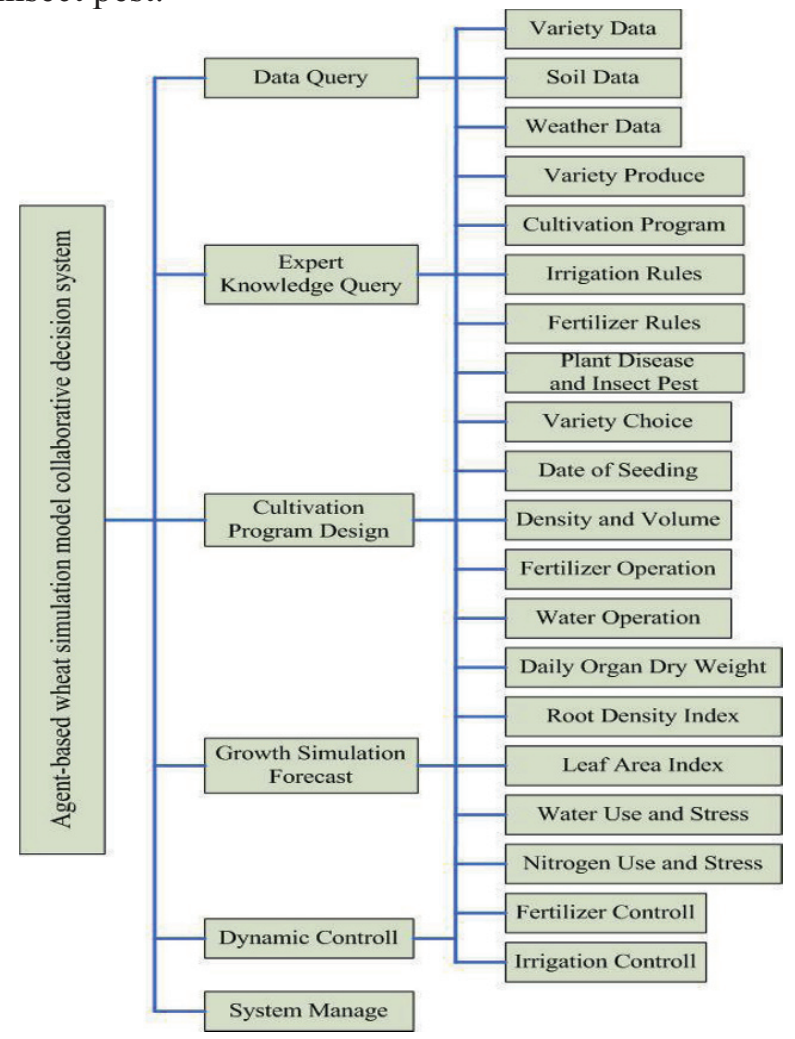

Fig. 1: system function diagram

\subsection{System Use Case Analysis}

In UML language use case describe system function from the system outside user point of view. When we analyze multi-agent system first of all we establish use case model according to their functional requirements $(\mathrm{Gu}$ Shaoyuan, and et al. 2001). Traditional use case diagram has two main objects: use case and actor. Multi-agent system is made of many agents with autonomy, sociality, reaction and initiative features, which are collaboration with each other and interact on users. In agent-oriented analysis and design we make some expansion in traditional UML and add agent in model as a 
new activities. Agent-based wheat simulation model collaborative decision system specific includes data manage agent, knowledge manage agent, knowledge model agent, growth model agent and dynamic control agent. Not only as part of the system, but also as independent users and administrators, they achieve interactive collaboration and cooperative work, help realizing system function and completing customer needs.

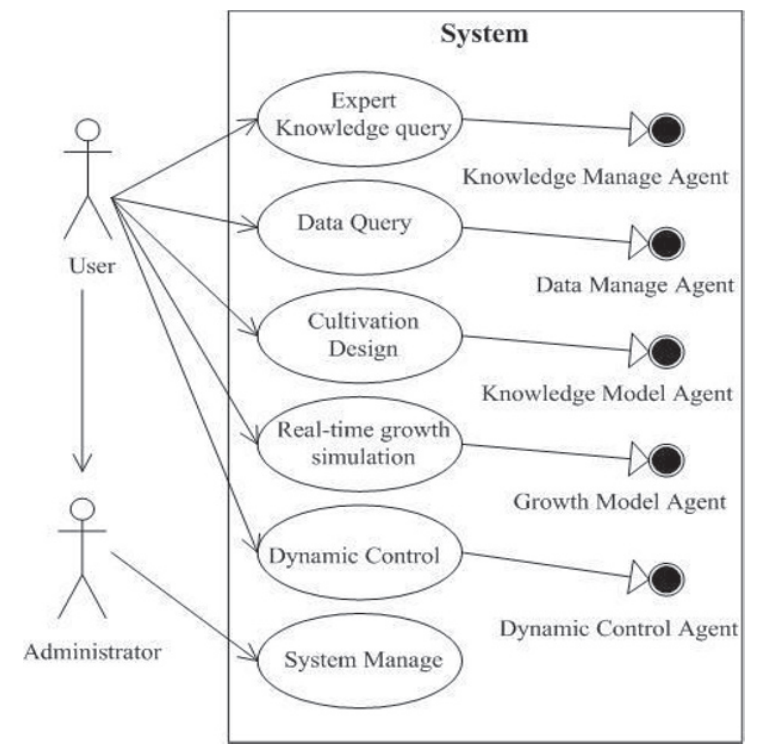

Fig.2: system use case diagram

\subsection{System Role Analysis}

In current agent-oriented method research, some researchers introduce role concept and regard role as basic unit of multi-agent system modeling. Compared with specific agent, role abstracts a common character of some agent. In multi-agent system, system object is assumed by every role agent (Ma Jun, Yan qi and et al. 2004). Making role as basic modeling unlit reflects goal-driven thinking of system modeling process, role have very important effect from analysis to the entire development process.

Agent-based wheat simulation model collaborative decision system designs 9 kind of role agent at four layers. At user layer we design Interface Agent. At resource layer we design Data Manage Agent, Knowledge Manage Agent and Model agent. Among Model Agent role agent registers as Knowledge Model Agent and Growth Model Agent, which belong to same role and complete different function. At decision layer role agent includes Decision Agent, Forecast Agent and Object Agent. At agent service layer role agent includes Communication Agent and Collaboration Agent. 


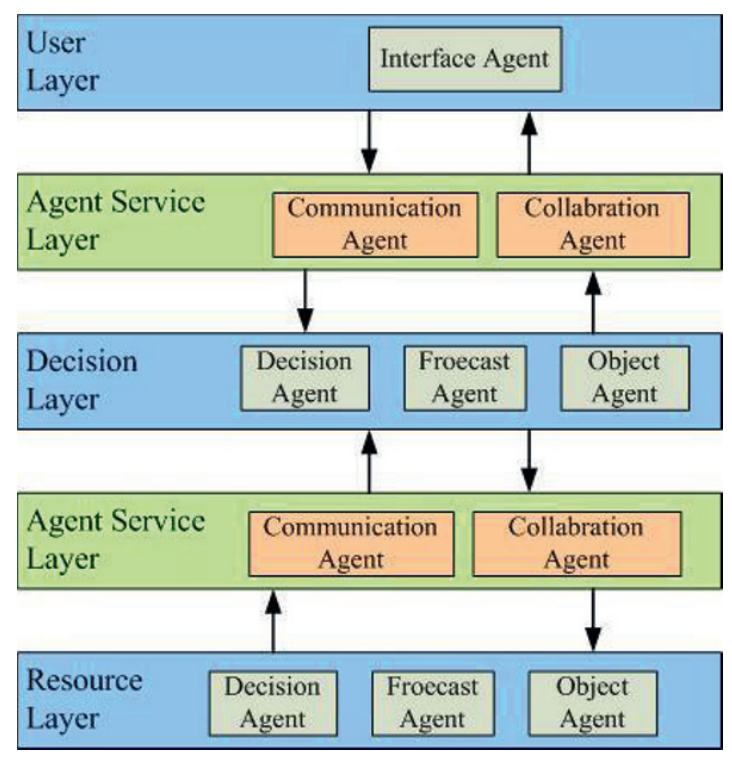

Fig.3: role layer diagram

\subsection{Agent Collaboration Analysis}

Interactive collaboration and cooperative work are important reflection of sociality, and also one of major advantages of Multi-Agent system. They express the interactive and dynamic cooperative relationship between Agents by cooperation diagram. Take achieving cultivation programs designing function in agent-based wheat simulation model collaborative decision system on the basis of MAS for an example, explain the interactive and cooperative relationship between Agents in the system.

When users need cultivation programs, firstly, put forward the task requests to Interface Agent which will send the task to Object Agent by calling Communication Agent. After Object Agent receives information and analyzes it, it will send messages to Data Manage Agent and pick up relevant data. Data manage agent will look up the perennial data on the decision-making point such as weather, soil and breeds etc. according to object agent's command, and send to Knowledge Model Agent which can run relevant models to produce a set of cultivation programs including breeds-choosing, sowing time, density, sowing quantity, fertilizer planning and moisture planning programs etc.. 


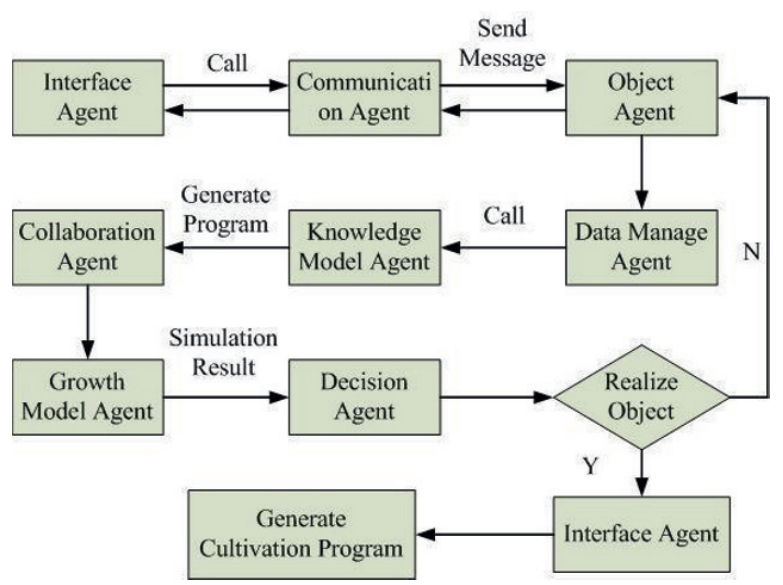

Fig.4: Collaboration diagram

And then Knowledge Model Agent will call Collaboration Agent, by which Knowledge Model Agent will transfer cultivation programs and the perennial data on the decision-making point such as weather, soil and breeds etc. produced by itself to Growth Model Agent. Growth Model Agent will simulate growth according to the information, and predict the yield and quality indexes gained by cultivation programs produced by carrying into Knowledge Model Agent, and send the prediction result to Decision Agent. Decision Agent will analyze the prediction result. If the yield and quality indexes predicted by Growth Model agent achieve the aim which users request, the result will be send back to Interface Agent, and export a set of cultivation programs. If not, the result will be send back to Object Agent and Interface Agent. After amending the aim program, simulate and predict, recycle as this until create a set of cultivation programs satisfying the demand.

\section{PROGRAM INTEGRATION AND DEVELOPMENT}

System development and operation choose Windows platform. To assure cross-platform commonality of multi-agent system and easy portable we choose JAVA language. We construct a number of different functions role agent through agent development and software integration. The wheat growth simulation, knowledge management, and other functional modules can fully use existing software programs for encapsulation and call so that we can construct the corresponding functional agent, save development workload and realize reusing and sharing of existing resources. 
Communication of multi-agent is built on the TCP/IP protocol. System platform environment includes a server agent and some client agent, server agent automatically start and monitor system after system start up. When all client agents start system first find server agent and register in it, at the same time, server agent can examine all register agent and manage life cycle of client agent which includes creating new agent, deleting an agent, suspending an agent and sending message to an agent.

Agent communication use currently the most popular agent communication language-KQML (Knowledge Query Manipulation Language). Specific sending mechanism exist difference with different location of Receive Agent. If Receive Agent and Send Agent in the same system, server agent directly process, send message to Receive Agent, if not, system need process message through agent communication channel in agent platform.

\section{CONCLUSION}

This research aims to improve the unilateralism of traditional crop growth simulation model system. It discusses integration mechanism for crop growth simulation model and knowledge model under Multi-Agent environment, pointing out crop management cooperation decision system based on Agent UML, analyzing and designing through several angle like function usage and cooperation decision and argument system Integration and development. Multi-Agent is intelligent and cooperative through analyzing the practice, which offers new technology means and methods for solving complicated problems of agriculture system.

Setting up Multi-Agent system platform is a new idea to integrated utilize current unilateral agriculture information system, meanwhile, it realizes resource share and cooperation decision, as well as improves information usage and management decision accuracy.

\section{ACKNOWLEDGEMENTS}

This research was supported by Digital Agriculture Program of State High-tech Research and Development Project of China (No. 2006AA10Z220, 2007AA10Z237), Special Fund of Basic Scientific Research and Operation Foundation for Commonweal Scientific Research Institutes (2008J-1-10, 2008J-1-02) and by National Scientific and Technical Supporting Programs Funded by Ministry of Science and Technology of China (2006BAD10A12). 


\section{REFERENCES}

CHEN Ying-chun, 2003. Intelligent decision support system based on Multi-Agent. Journal of Hefei University of Technologic (Social Sciences), Vol. 17 No.6

DeLoach, S.A. 2006. Engineering Organization-based Multiagent Systems. LNCS Vol. 3914, Springer, 109-125

DING Wei-long, 2005. Research of the agricultural expert system based on artificial plant growth model. Journal of Zhejiang University of Technology, Vol. 27 , Supp. 2

F. Bousquet and et al. 2002. Multi-agent systems and role games: collective learning processes for ecosystem management. In Complexity and ecosystem management: The theory and practice of multi-agent systems, pages 248 \{285. Edward Elgar.

Gu Shaoyuan, Zhu Chenchen, Shi Hongbao,2001. A Agent-Based Method for Requirement Analysis and Modeling. Computer Engineering and Applications, Vol.5

M. Wooldridge, N. Jennings, and D. Kinny. 2000. The Gaia Methodology for Agent-Oriented Analysis and Design. Journal of Autonomous Agents and Multi-Agent Systems, 3(3).

Ma Jun, Yan qi and et al. 2004, Role-Based Software Design Method for Multi-Agent System. Computer Engineering and Applications, Vol.6

N.HARNOS. 2006. Applicability of the AFRCWHEAT2 wheat growth simulation model in Hungary. APPLIED ECOLOGY AND ENVIRONMENTAL RESEARCH 4(2): 55-61.

WENG Wen-yong, WANG Ze-bing, FENG Yan, 2004. Research on applying UML to analyze agent-oriented system. Computer Engineering and Design, Vol.25, No.7

ZHU Ye-ping, FENG Zhong-ke, 2005. Application of agent in agricultural \& forestry economy decision support system. Journal of Beijing Forestry University, Vol. 27 , Supp. 2 